\title{
Determination of Lanthanides in Soil and Sediment Reference Samples by ICP-MS: Comparison of Fusion and Acid Digestion Methods
}

\author{
Manik C. Saha*, Sudipta Lahiri, Rashmoni Baskey, and F. Kharshiing \\ Geological Survey of India, Central Headquarters, Chemical Division, \\ 15 A \& B Kyd Street, Kolkata 700 016, India
}

\section{INTRODUCTION}

There are 15 rare earth elements (REEs) with atomic numbers from 57 through 71 of which one element, promethium $(\mathrm{Pm})$, is radioactive and does not exist in nature. REEs are found in many soils in concentrations of up to tens of parts per million with organic soils usually richer in lanthanides than mineral soils (1). Traditionally, REEs are divided into two sub groups: light rare earth elements (LREEs) from La to Eu and heavy rare earth elements (HREEs) from Gd to $\mathrm{Lu}(2-3)$.

Soil erosion is a global environmental problem which is often easy to observe but hard to measure. One monitoring technique is tagging of soil with tracers. The REE oxides have been successfully used as a multi-sediment tracers (4-6). REEs have also been used to explain many geochemical processes in the environment, such as natural surface weathering processes, origin of some sediments, identification of anthropogenic impact, and palaeo-environmental and palaeo-oceanic changes (3, 7-15). The importance of REEs in geochemistry for modelling the earth's crustal and mantle evolutionary processes and dating the age of rocks is beyond any doubt.

Samples are brought into solution before their determination by instrumental methods. Complete dissolution is a fundamental factor for obtaining precise and accurate analytical data (16). Fusion and acid digestion with different combina-

*Corresponding authors.

E-mail: manikchandra.saba@yaboo.com

\section{ABSTRACT}

The determination of lanthanides in six soil and six sediment reference materials was investigated by inductively coupled plasma mass spectrometry (ICPMS) using fusion and acid digestion. In the present study, the samples were fused with lithium metaborate $\left(\mathrm{LiBO}_{2}\right)$ and lithium tetraborate $\left(\mathrm{Li}_{2} \mathrm{~B}_{4} \mathrm{O}_{7}\right)$. In the case of acid decomposition, the samples were digested with a mixture of hydrofluoric acid (HF) and perchloric acid $\left(\mathrm{HClO}_{4}\right)$. For both cases, 1:1 $\mathrm{HNO}_{3}$ was used for extraction of the decomposed samples. The internal standard (10 ppb indium) was used to take care of the signal drift of the instrument. The measurement was done using a 1 ppb matrixmatched calibration standard prepared from lanthanide salts. The effect of different combinations of fusion flux was investigated. A detailed study was also made taking a different isotopic mass for measurement of the lanthanides.

Both dissolution techniques were evaluated. Uniform and good recovery of the lanthanides was observed using a fusion mixture containing 35\% lithium tetraborate and $65 \%$ lithium metaborate. The accuracy of the methodology was excellent. Extraction of the lanthanides from the soil and sediment samples by acid digestion method varied from $80-105 \%$. Precision and accuracy in case of the fusion method was very good. The limit of quantitation $(10 \sigma)$ for the determination of the lanthanides in soil and sediment samples was in the ppb range. tions are normally adopted for complete dissolution of geological samples. Some workers have used open vessel acid digestion for dissolution of rock samples with or without fusion of any insoluble residue and then direct determination of the REEs by inductively coupled plasma mass spectrometry (ICP-MS) (17-19).

Because the crustal abundance of REEs is very low, pre-concentration procedures such as solvent extraction, co-precipitation or ionexchange separation are used by some workers to achieve a lower limit of detection (20-24). Fudyunina et al. (25) determined REEs in rock samples using lithium metaborate fusion followed by the pre-concentration on Pol-DETATA, and reported less than 15\% difference between the certified and observed values. The present authors used a mixture of lithium metaborate and lithium tetraborate which reduces the melting point of the fusion mixture and also dissolves the acidic and basic oxides easily (26). The pre-concentration procedures are time-consuming and labor-

intensive. Sengupta et al. (27) reported that direct ICP-MS determination of lanthanides in common rocks and sediments is possible with acceptable accuracy and precision after the decomposition of the samples by microwave digestion with acids and the complexation of excess $\mathrm{HF}$ and fluoride with boric acid and EDTA. Begum et al. (28) applied an open and closed vessel dissolution technique with $\mathrm{HF}$, $\mathrm{HClO}_{4}$ and $\mathrm{HNO}_{3}$ for the determination of REEs in marine sediment samples by ICP-MS and reported a better yield with closed vessel dissolution. Microwave digestion or closed vessel dissolution with a 
mixture of acids requires evaporation of $\mathrm{HF}$ and $\mathrm{HClO}_{4}$ or complexation of HF for the elemental determination by instrumental techniques.

The instrumental techniques used for the determination of REEs in rock samples are mainly neutron activation (NA), isotope dilution mass spectrometry, ICP-AES and ICP-MS, of which ICP-MS is the most widely used technique for the determination of REEs in different types of rock samples (29). It is a rapid multi-elemental detection technique with very low detection limits, high sensitivity, high sample throughput, fewer interferences, and a wide range of detection (30). The REEs in chondrite and meteorite were determined using the acid dissolution procedure and found ICP-MS to be a better and more precise measurement technique (31-32).

In the present study, a comparison of two dissolution methods, namely fusion and acid digestion, was made for the direct determination of REEs in soil and sediment reference materials and samples analyzed by ICP-MS. Six sediment and six soil reference samples were used to validate the analytical methodology for the determination of rare earth elements. There are very few studies available on the determination of REEs in soil and sediment samples, which requires an accurate and simple method for routine determination. The objective of the present study was to establish a simple, fast, precise, accurate, and less labor-intensive method for the determination of REEs in soil and sediment samples with detection limits much below the crustal abundance.

\section{EXPERIMENTAL}

\section{Instrumentation}

All analyses were performed using a model ELAN ${ }^{\circledR}$ DRC $^{\text {TM }}-\mathrm{e}$ ICP-MS instrument (PerkinElmer, Inc., Shelton, CT, USA). The instrumental parameters are listed in Table I. The data acquisition parameters and isotopes used for measurement are listed in Tables II and III, respectively.

TaABLE I

ICP-MS Instrumental Parameters

\begin{tabular}{ll|ll}
\hline RF Power & $1125 \mathrm{~W}$ & Plasma Gas Flow & $15.50 \mathrm{~L} / \mathrm{min}$ \\
Nebulizer Gas Flow & $0.85 \mathrm{~L} / \mathrm{min}$ & Lens Voltage & $7.75 \mathrm{~V}$ \\
Auxiliary Gas Flow & $1.20 \mathrm{~L} / \mathrm{min}$ & Sample Uptake Rate & $1.0 \mathrm{~mL} / \mathrm{min}$ \\
\hline
\end{tabular}

TABLE II

Data Acquisition Parameters

\begin{tabular}{ll|ll}
\hline Measuring Mode & Peak Hopping & Integration Time & $2500 \mathrm{~ms}$ \\
Points per Peak & 1 & Replicates & 3 \\
Number of Sweeps & 50 & Internal Standard & ${ }^{115} \mathrm{In}$ \\
Dwell Time & $50 \mathrm{~ms}$ & & \\
\hline
\end{tabular}

TABLE III

Isotopes for ICP-MS Analysis

\begin{tabular}{|c|c|c|c|c|c|c|c|c|c|c|c|c|c|c|}
\hline Element & $\mathrm{La}$ & $\mathrm{Ce}$ & $\operatorname{Pr}$ & $\mathrm{Nd}$ & $\mathrm{Sm}$ & $\mathrm{Eu}$ & Gd & $\mathrm{Tb}$ & Dy & Ho & $\mathrm{Er}$ & $\mathrm{Tm}$ & $\mathrm{Yb}$ & $\mathrm{Lu}$ \\
\hline Mass & 136. & 139. & 140. & 142. & 146. & 150. & 157. & 158. & 162. & 164. & 166. & 168. & 171. & 174. \\
\hline Number & 906 & 905 & 907 & 910 & 915 & 920 & 924 & 925 & 925 & 930 & 932 & 930 & 937 & 941 \\
\hline
\end{tabular}


and six soil reference samples of GSS-1, GSS-2, GSS-3, GSS-5, GSS-6, and GSS-7.

\section{Sample Dissolution}

\section{Acid Digestion Method}

A quantity of $0.2 \mathrm{~g}$ sample was accurately weighed into a Teflon ${ }^{\circledR}$ beaker, to which $8 \mathrm{~mL} \mathrm{HF}$ and $3 \mathrm{~mL}$ $\mathrm{HClO}_{4}$ were added. The beaker was covered with a lid and heated slowly on hot plate for one hour to about $110{ }^{\circ} \mathrm{C}$. The lid was removed to evaporate HF. Then the sample was heated to about $220^{\circ} \mathrm{C}$ under covered conditions for about one hour to obtain a clear solution. The solution was then evaporated to a pasty mass. The residue was dissolved by heating with $30 \mathrm{~mL} \mathrm{8 \%}$ $\mathrm{HNO}_{3}$. After cooling to room temperature, the volume was made up to $100 \mathrm{~mL}$ in a volumetric flask. The indium concentration of the solution was maintained at $10 \mathrm{ppb}$ using $2 \mathrm{~mL}$ of $500 \mathrm{ppb}$ In solution which acts as internal standard. Procedural blanks were prepared similarly

\section{Fusion Metbod}

To start, $0.1 \mathrm{~g}$ of sample, $0.15 \mathrm{~g}$ of $\mathrm{Li}_{2} \mathrm{~B}_{4} \mathrm{O}_{7}$, and $0.2 \mathrm{~g}$ of $\mathrm{LiBO}_{2}$ were accurately weighed into a platinum crucible and mixed homogeneously. It was then placed into a muffle furnace and heated in stages up to $1000{ }^{\circ} \mathrm{C}$ for two and half hours. The heating rate was $6^{\circ} \mathrm{C} / \mathrm{min}$ up to $250^{\circ} \mathrm{C}$, then $7{ }^{\circ} \mathrm{C} / \mathrm{min}$ up to $700{ }^{\circ} \mathrm{C}$. and finally $6{ }^{\circ} \mathrm{C} / \mathrm{min}$ up to $1000{ }^{\circ} \mathrm{C}$, allowing 10 minutes resi- dency period at each stage. After cooling to room temperature, the oily melt residue obtained was dissolved with $30 \mathrm{~mL} 8 \% \mathrm{HNO}_{3}$ by stirring at $720 \mathrm{rpm}$ for 30 minutes with a Teflon ${ }^{\circledR}$-coated magnetic bar until a clear solution was obtained. The volume was made up to $250 \mathrm{~mL}$ in a volumetric flask. The indium concentration in the solution was maintained at $10 \mathrm{ppb}$ using $5 \mathrm{~mL}$ of 500 ppb In solution which acts as internal standard. Procedural blanks were prepared similarly.

\section{RESULTS AND DISCUSSION}

This work analyzed 12 certified reference materials using both dissolution techniques for comparative study. Indium was added as an internal standard to take care of the signal drift of the instrument caused by changes in nebulizer efficiency, gradual clogging of the injector and cones, and matrixinduced suppression and enhancement effects. Spectroscopic interferences arise mainly due to isobaric overlaps which have been minimized by choosing an isotope having minimum isobaric interferences and applying isobaric correction factors, when required. Of the 14 lanthanides, $\mathrm{Pr}, \mathrm{Tb}$, Ho, and Tm consist of only a single isotopic mass, whereas for $\mathrm{La}, \mathrm{Eu}$, and $\mathrm{Lu}$ two isotopes exist. The isotopic ratio for $\mathrm{Eu}$ is almost 1 , but in the case of $\mathrm{La}$ and $\mathrm{Lu}$, only one isotope is predominant. For the rest of the seven elements, the isotope was selected based on the natural abun-

TABLE IV

Elements, Compounds, and Weight Taken for 1000 ppm Lanthanide Solution

\begin{tabular}{lccccccc}
\hline Elements & $\mathrm{La}$ & $\mathrm{Ce}$ & $\mathrm{Pr}$ & $\mathrm{Nd}$ & $\mathrm{Eu}$ & $\mathrm{Sm}$ & $\mathrm{Gd}$ \\
\hline Compounds & $\mathrm{La}_{2} \mathrm{O}_{3}$ & $\mathrm{Ce}_{2} \mathrm{O}_{3}$ & $\mathrm{Pr}_{2} \mathrm{O}_{3}$ & $\mathrm{Nd}_{2} \mathrm{O}_{3}$ & $\mathrm{Eu}_{2} \mathrm{O}_{3}$ & $\mathrm{Sm}_{2} \mathrm{O}_{3}$ & $\mathrm{Gd}_{2} \mathrm{O}_{3}$ \\
Weight $(\mathrm{g})$ & 0.5864 & 0.6142 & 0.6041 & 0.5832 & 0.5790 & 0.5798 & 0.5763 \\
\hline Elements & $\mathrm{Tb}$ & $\mathrm{Dy}$ & $\mathrm{Ho}$ & $\mathrm{Er}$ & $\mathrm{Tm}$ & $\mathrm{Yb}$ & $\mathrm{Lu}$ \\
\hline Compounds & $\mathrm{Tb}_{4} \mathrm{O}_{7}$ & $\mathrm{Dy}_{2} \mathrm{O}_{3}$ & $\mathrm{Ho}_{2} \mathrm{O}_{3}$ & $\mathrm{Er}_{2} \mathrm{O}_{3}$ & $\mathrm{Tm}_{2} \mathrm{O}_{3}$ & $\mathrm{Yb}_{2} \mathrm{O}_{3}$ & $\mathrm{Lu}_{2} \mathrm{O}_{3}$ \\
Weight (g) & 0.5881 & 0.5738 & 0.5717 & 0.2858 & 0.5693 & 0.5685 & 0.6349 \\
\hline
\end{tabular}


TABLE V(A)

Results of Stream Sediment Samples

\begin{tabular}{|c|c|c|c|c|c|c|c|c|c|}
\hline \multirow{2}{*}{$\begin{array}{c}\text { Ele- } \\
\text { ments }\end{array}$} & \multicolumn{3}{|c|}{ CRM GSD-4 } & \multicolumn{3}{|c|}{ CRM GSD- 5} & \multicolumn{3}{|c|}{ CRM GSD- 6} \\
\hline & A & B & $\mathrm{C}$ & A & B & $\mathrm{C}$ & A & B & $\mathrm{C}$ \\
\hline $\mathrm{La}$ & 42.03 & 42.23 & $40 \pm 9$ & 39.72 & 44.11 & $46 \pm 7$ & 33.00 & 40.79 & $39 \pm 8$ \\
\hline $\mathrm{Ce}$ & 73.02 & 77.57 & $78 \pm 4$ & 74.15 & 85.34 & $89 \pm 8$ & 55.01 & 65.95 & $68 \pm 8$ \\
\hline $\operatorname{Pr}$ & 8.68 & 9.37 & $9.3 \pm 1.3$ & 8.47 & 9.43 & $9.9 \pm 0.9$ & 7.18 & 8.72 & $8.4 \pm 0.6$ \\
\hline $\mathrm{Nd}$ & 33.69 & 36.40 & $32 \pm 4$ & 32.40 & 37.24 & $35 \pm 5$ & 28.69 & 34.78 & $33 \pm 6$ \\
\hline $\mathrm{Sm}$ & 5.51 & 6.40 & $6.2 \pm 0.5$ & 5.26 & 5.81 & $6.6 \pm 0.5$ & 4.67 & 5.70 & $5.6 \pm 0.6$ \\
\hline $\mathrm{Eu}$ & 1.23 & 1.25 & $1.31 \pm 0.13$ & 1.20 & 1.29 & $1.4 \pm 0.2$ & 1.30 & 1.51 & $1.5 \pm 0.13$ \\
\hline Gd & 5.81 & 6.07 & $5.0 \pm 0.8$ & 5.79 & 7.75 & $6.4 \pm 1.1$ & 5.05 & 5.35 & $5.5 \pm 0.9$ \\
\hline $\mathrm{Tb}$ & 0.63 & 0.86 & $0.90 \pm 0.16$ & 0.59 & 0.84 & $0.89 \pm 0.17$ & 0.53 & 0.73 & $0.69 \pm 0.15$ \\
\hline Dy & 3.24 & 5.19 & $4.6 \pm 0.4$ & 2.90 & 4.99 & $5 \pm 0.5$ & 2.60 & 4.04 & $3.8 \pm 1$ \\
\hline Ho & 0.64 & 0.84 & $1.0 \pm 0.2$ & 0.58 & 0.81 & $0.95 \pm 0.15$ & 0.52 & 0.66 & $0.76 \pm 0.12$ \\
\hline $\mathrm{Er}$ & 1.68 & 2.70 & $2.5 \pm 0.4$ & 1.48 & 2.91 & $2.8 \pm 0.5$ & 1.32 & 2.25 & $2.2 \pm 0.5$ \\
\hline $\mathrm{Tm}$ & 0.23 & 0.39 & $0.46 \pm 0.05$ & 0.20 & 0.38 & $0.46 \pm 0.05$ & 0.17 & 0.29 & $0.35 \pm 0.07$ \\
\hline $\mathrm{Yb}$ & 1.67 & 3.08 & $2.9 \pm 0.4$ & 1.47 & 2.88 & $2.9 \pm 0.3$ & 1.22 & 2.17 & $2.1 \pm 0.4$ \\
\hline $\mathrm{Lu}$ & 0.25 & 0.44 & $0.47 \pm 0.13$ & 0.22 & 0.41 & $0.46 \pm 0.05$ & 0.18 & 0.32 & $0.34 \pm 0.08$ \\
\hline
\end{tabular}

A: Acid Digestion Method.

B: Fusion Method

C: Certified Values

TABLE V(B)

Results of Stream Sediment Samples

\begin{tabular}{|c|c|c|c|c|c|c|c|c|c|}
\hline \multirow{2}{*}{$\begin{array}{c}\text { Ele- } \\
\text { ments }\end{array}$} & \multicolumn{3}{|c|}{ CRM GSD-7 } & \multicolumn{3}{|c|}{ CRM GSD-10 } & \multicolumn{3}{|c|}{ CRM GSD-11 } \\
\hline & A & B & C & A & $\mathrm{B}$ & $\mathrm{C}$ & A & B & $\mathrm{C}$ \\
\hline $\mathrm{La}$ & 40.02 & 46.07 & $45 \pm 6$ & 11.58 & 13.22 & $13 \pm 1.4$ & 31.30 & 27.88 & $30 \pm 3$ \\
\hline $\mathrm{Ce}$ & 65.80 & 79.56 & $78 \pm 7$ & 29.87 & 38.76 & $38 \pm 5$ & 52.37 & 53.90 & $58 \pm 5$ \\
\hline $\operatorname{Pr}$ & 8.84 & 9.94 & $9.6 \pm 1.1$ & 2.53 & 2.93 & $3.2 \pm 0.4$ & 6.99 & 6.09 & $7.4 \pm 0.6$ \\
\hline $\mathrm{Nd}$ & 32.06 & 37.86 & $37 \pm 6$ & 10.50 & 11.76 & $11.8 \pm 1.6$ & 27.44 & 26.18 & $27 \pm 3$ \\
\hline $\mathrm{Sm}$ & 5.06 & 5.57 & $6.1 \pm 0.5$ & 1.86 & 2.40 & $2.4 \pm 0.2$ & 5.76 & 5.60 & $6.2 \pm 0.4$ \\
\hline $\mathrm{Eu}$ & 1.13 & 1.30 & $1.3 \pm 0.2$ & 0.38 & 0.44 & $0.47 \pm 0.05$ & 0.54 & 0.58 & $0.6 \pm .08$ \\
\hline Gd & 5.19 & 6.03 & $5.8 \pm 0.8$ & 2.34 & 2.50 & $2.2 \pm 0.3$ & 6.35 & 7.52 & $5.9 \pm 0.5$ \\
\hline $\mathrm{Tb}$ & 0.58 & 0.77 & $0.76 \pm 0.14$ & 0.31 & 0.38 & $0.42 \pm 0.11$ & 0.83 & 1.04 & $1.13 \pm 0.1$ \\
\hline Dy & 2.99 & 4.56 & $4.2 \pm 0.7$ & 1.84 & 2.27 & $2.2 \pm 0.3$ & 5.16 & 7.31 & $7.2 \pm 0.8$ \\
\hline Ho & 0.60 & 0.72 & $0.96 \pm 0.21$ & 0.34 & 0.38 & $0.45 \pm 0.08$ & 0.97 & 1.21 & $1.4 \pm 0.2$ \\
\hline $\mathrm{Er}$ & 1.61 & 2.65 & $2.3 \pm 0.2$ & 1.08 & 1.34 & $1.3 \pm 0.2$ & 3.05 & 4.65 & $4.6 \pm 0.6$ \\
\hline $\mathrm{Tm}$ & 0.22 & 0.34 & $0.44 \pm 0.09$ & 0.14 & 0.17 & $0.2 \pm 0.04$ & 0.43 & 0.66 & $0.74 \pm 0.11$ \\
\hline $\mathrm{Yb}$ & 1.53 & 2.58 & $2.6 \pm 0.4$ & 0.98 & 1.28 & $1.2 \pm 0.3$ & 3.47 & 5.11 & $5.1 \pm 0.8$ \\
\hline $\mathrm{Lu}$ & 0.24 & 0.44 & $0.39 \pm 0.07$ & 0.14 & 0.18 & $0.19 \pm 0.04$ & 0.46 & 0.77 & $0.78 \pm 0.08$ \\
\hline
\end{tabular}

A: Acid Digestion Method.

B: Fusion Method.

C: Certified Values. 


\section{Atomic Spectroscopy \\ $\bigcirc$ Vol. 37(5), Sept./Oct. 2016}

Table VI(A)

Results of Soil Samples

\begin{tabular}{|c|c|c|c|c|c|c|c|c|c|}
\hline \multirow{2}{*}{$\begin{array}{c}\text { Ele- } \\
\text { ments }\end{array}$} & \multicolumn{3}{|c|}{ CRM GSS-1 } & \multicolumn{3}{|c|}{ CRM GSS-2 } & \multicolumn{3}{|c|}{ CRM GSS-3 } \\
\hline & A & B & $\mathrm{C}$ & A & $\mathrm{B}$ & $\mathrm{C}$ & A & B & $\mathrm{C}$ \\
\hline $\mathrm{La}$ & 37.80 & 35.84 & $34 \pm 3$ & 148.49 & 160.22 & $164 \pm 16$ & 20.17 & 21.31 & $21 \pm 2$ \\
\hline $\mathrm{Ce}$ & 72.02 & 71.64 & $70 \pm 5$ & 385.09 & 408.67 & $402 \pm 25$ & 37.44 & 39.39 & $39 \pm 6$ \\
\hline $\operatorname{Pr}$ & 8.04 & 7.32 & $7.5 \pm 0.5$ & 51.22 & 57.53 & $57 \pm 6$ & 4.67 & 4.68 & $4.8 \pm 0.4$ \\
\hline $\mathrm{Nd}$ & 30.52 & 29.15 & $28 \pm 3$ & 196.30 & 224.68 & $210 \pm 22$ & 18.10 & 17.26 & $18.4 \pm 2.4$ \\
\hline $\mathrm{Sm}$ & 5.35 & 5.09 & $5.2 \pm 0.4$ & 15.24 & 18.23 & $18 \pm 3$ & 2.79 & 2.85 & $3.3 \pm 0.3$ \\
\hline $\mathrm{Eu}$ & 1.04 & 0.97 & $1.0 \pm 0.1$ & 2.87 & 2.95 & $3.0 \pm 0.3$ & 0.75 & 0.70 & $0.72 \pm 0.06$ \\
\hline Gd & 5.00 & 4.41 & $4.6 \pm 0.3$ & 8.45 & 8.67 & $7.8 \pm 0.6$ & 2.85 & 2.80 & $2.9 \pm 0.4$ \\
\hline $\mathrm{Tb}$ & 0.73 & 0.80 & $0.75 \pm 0.09$ & 1.06 & 1.28 & $0.97 \pm 0.40$ & 0.35 & 0.45 & $0.49 \pm 0.09$ \\
\hline Dy & 3.74 & 4.32 & $4.6 \pm 0.3$ & 3.65 & 4.33 & $4.4 \pm 0.3$ & 1.91 & 2.45 & $2.6 \pm 0.2$ \\
\hline Ho & 0.75 & 0.91 & $0.87 \pm 0.08$ & 0.67 & 0.85 & $0.93 \pm 0.15$ & 0.38 & 0.44 & $0.53 \pm 0.07$ \\
\hline $\mathrm{Er}$ & 1.86 & 2.52 & $2.6 \pm 0.2$ & 1.45 & 2.19 & $2.1 \pm 0.4$ & 1.09 & 1.49 & $1.5 \pm 0.3$ \\
\hline $\mathrm{Tm}$ & 0.29 & 0.45 & $0.42 \pm 0.07$ & 0.19 & 0.33 & $0.42 \pm 0.13$ & 0.17 & 0.22 & $0.28 \pm 0.06$ \\
\hline $\mathrm{Yb}$ & 1.90 & 2.59 & $2.7 \pm 0.4$ & 1.27 & 2.11 & $2.0 \pm 0.3$ & 1.10 & 1.76 & $1.7 \pm 0.3$ \\
\hline $\mathrm{Lu}$ & 0.30 & 0.44 & $0.41 \pm 0.06$ & 0.20 & 0.31 & $0.32 \pm 0.06$ & 0.17 & 0.26 & $0.29 \pm 0.03$ \\
\hline
\end{tabular}

A: Acid Digestion Method.

B: Fusion Method.

C: Certified Values.

Table VI(B)

Results of Soil Samples

\begin{tabular}{|c|c|c|c|c|c|c|c|c|c|}
\hline \multirow{2}{*}{$\begin{array}{l}\text { Ele- } \\
\text { ments }\end{array}$} & \multicolumn{3}{|c|}{ CRM GSS-5 } & \multicolumn{3}{|c|}{ CRM GSS-6 } & \multicolumn{3}{|c|}{ CRM GSS-7 } \\
\hline & A & B & $\mathrm{C}$ & A & B & $\mathrm{C}$ & A & B & $\mathrm{C}$ \\
\hline $\mathrm{La}$ & 32.95 & 32.77 & $36 \pm 6$ & 29.54 & 29.96 & $30 \pm 3$ & 39.17 & 43.16 & $46 \pm 7$ \\
\hline $\mathrm{Ce}$ & 88.81 & 85.04 & $91 \pm 15$ & 57.43 & 60.27 & $66 \pm 8$ & 90.16 & 92.12 & $98 \pm 16$ \\
\hline $\operatorname{Pr}$ & 6.43 & 6.50 & $7 \pm 1.3$ & 5.90 & 5.77 & $5.8 \pm 0.6$ & 9.81 & 10.17 & $11 \pm 1$ \\
\hline $\mathrm{Nd}$ & 23.01 & 23.78 & $24 \pm 2$ & 21.52 & 21.03 & $21 \pm 3$ & 40.69 & 41.48 & $45 \pm 3$ \\
\hline $\mathrm{Sm}$ & 3.62 & 3.90 & $4 \pm 0.6$ & 3.34 & 4.28 & $3.8 \pm 0.6$ & 8.79 & 9.14 & $10.3 \pm 0.6$ \\
\hline $\mathrm{Eu}$ & 0.75 & 0.68 & $0.82 \pm 0.06$ & 0.62 & 0.64 & $0.66 \pm 0.06$ & 3.32 & 3.41 & $3.4 \pm 0.3$ \\
\hline Gd & 3.57 & 3.48 & $3.5 \pm 0.3$ & 3.04 & 3.57 & $3.4 \pm 0.3$ & 8.66 & 9.64 & $9.6 \pm 1$ \\
\hline $\mathrm{Tb}$ & 0.47 & 0.58 & $0.7 \pm 0.2$ & 0.44 & 0.53 & $0.61 \pm 0.12$ & 0.87 & 1.13 & $1.3 \pm 0.3$ \\
\hline Dy & 2.55 & 3.16 & $3.7 \pm 0.6$ & 2.65 & 3.30 & $3.3 \pm 0.3$ & 4.67 & 6.26 & $6.6 \pm 0.8$ \\
\hline Ho & 0.53 & 0.68 & $0.8 \pm 0.2$ & 0.48 & 0.64 & $0.69 \pm 0.06$ & 0.69 & 0.83 & $1.1 \pm 0.2$ \\
\hline $\mathrm{Er}$ & 1.82 & 2.25 & $2.4 \pm 0.3$ & 1.68 & 2.20 & $2.2 \pm 0.3$ & 1.87 & 2.61 & $2.7 \pm 0.6$ \\
\hline $\mathrm{Tm}$ & 0.29 & 0.38 & $0.41 \pm 0.05$ & 0.28 & 0.31 & $0.40 \pm 0.07$ & 0.20 & 0.32 & $0.42 \pm 0.06$ \\
\hline $\mathrm{Yb}$ & 1.87 & 2.43 & $2.8 \pm 0.5$ & 1.98 & 2.65 & $2.7 \pm 0.5$ & 1.54 & 2.27 & $2.4 \pm 0.6$ \\
\hline $\mathrm{Lu}$ & 0.27 & 0.37 & $0.42 \pm 0.07$ & 0.30 & 0.41 & $0.42 \pm 0.06$ & 0.19 & 0.29 & $0.35 \pm 0.08$ \\
\hline
\end{tabular}

A: Acid Digestion Method.

B: Fusion Method.

C: Certified Values. 
TABLE VII(A)

Percent Recovery of Stream Sediment Samples

\begin{tabular}{|c|c|c|c|c|c|c|c|c|c|c|c|c|}
\hline \multirow{2}{*}{$\begin{array}{c}\text { Ele- } \\
\text { ments }\end{array}$} & \multicolumn{2}{|c|}{ CRM GSD-4 } & \multicolumn{2}{|c|}{ CRM GSD-5 } & \multicolumn{2}{|c|}{ CRM GSD-6 } & \multicolumn{2}{|c|}{ CRM GSD-7 } & \multicolumn{2}{|c|}{ CRM GSD-10 } & \multicolumn{2}{|c|}{ CRM GSD-11 } \\
\hline & A & B & A & B & A & B & A & B & A & $\mathrm{B}$ & A & $\mathrm{B}$ \\
\hline $\mathrm{La}$ & 105 & 106 & 86 & 96 & 85 & 105 & 89 & 102 & 89 & 102 & 104 & 93 \\
\hline $\mathrm{Ce}$ & 94 & 99 & 83 & 96 & 81 & 97 & 84 & 102 & 79 & 102 & 90 & 93 \\
\hline $\operatorname{Pr}$ & 93 & 101 & 86 & 95 & 85 & 104 & 92 & 104 & 79 & 92 & 94 & 82 \\
\hline $\mathrm{Nd}$ & 105 & 114 & 93 & 106 & 87 & 105 & 87 & 102 & 89 & 100 & 102 & 97 \\
\hline $\mathrm{Sm}$ & 89 & 103 & 80 & 88 & 83 & 102 & 83 & 91 & 78 & 100 & 93 & 90 \\
\hline $\mathrm{Eu}$ & 94 & 95 & 86 & 92 & 87 & 101 & 87 & 100 & 81 & 94 & 90 & 97 \\
\hline Gd & 116 & 121 & 90 & 121 & 92 & 97 & 89 & 104 & 106 & 114 & 108 & 127 \\
\hline $\mathrm{Tb}$ & 70 & 96 & 66 & 94 & 77 & 106 & 76 & 101 & 74 & 90 & 73 & 92 \\
\hline Dy & 70 & 113 & 58 & 100 & 68 & 106 & 71 & 109 & 84 & 103 & 72 & 102 \\
\hline Ho & 64 & 84 & 61 & 85 & 68 & 87 & 63 & 75 & 76 & 84 & 69 & 86 \\
\hline $\mathrm{Er}$ & 67 & 108 & 53 & 104 & 60 & 102 & 70 & 115 & 83 & 103 & 66 & 101 \\
\hline $\mathrm{Tm}$ & 50 & 85 & 43 & 83 & 49 & 83 & 50 & 77 & 70 & 85 & 58 & 89 \\
\hline $\mathrm{Yb}$ & 58 & 106 & 51 & 99 & 58 & 103 & 59 & 99 & 82 & 107 & 68 & 100 \\
\hline $\mathrm{Lu}$ & 53 & 94 & 48 & 89 & 53 & 94 & 62 & 113 & 74 & 95 & 59 & 99 \\
\hline
\end{tabular}

A: Acid Digestion Method.

B: Fusion Method.

TABLE VII(B)

Percent Recovery of Soil Samples

\begin{tabular}{|c|c|c|c|c|c|c|c|c|c|c|c|c|}
\hline \multirow{2}{*}{$\begin{array}{c}\text { Ele- } \\
\text { ments }\end{array}$} & \multicolumn{2}{|c|}{ CRM GSS-1 } & \multicolumn{2}{|c|}{ CRM GSS-2 } & \multicolumn{2}{|c|}{ CRM GSS-3 } & \multicolumn{2}{|c|}{ CRM GSS-5 } & \multicolumn{2}{|c|}{ CRM GSS-6 } & \multicolumn{2}{|c|}{ CRM GSS-7 } \\
\hline & A & B & A & B & A & B & A & B & A & B & A & B \\
\hline $\mathrm{La}$ & 111 & 105 & 91 & 98 & 96 & 101 & 92 & 91 & 98 & 100 & 85 & 94 \\
\hline $\mathrm{Ce}$ & 103 & 102 & 96 & 102 & 96 & 101 & 98 & 93 & 87 & 91 & 92 & 94 \\
\hline $\operatorname{Pr}$ & 107 & 98 & 90 & 101 & 97 & 98 & 92 & 93 & 102 & 99 & 89 & 92 \\
\hline $\mathrm{Nd}$ & 109 & 104 & 93 & 107 & 98 & 94 & 96 & 99 & 102 & 100 & 90 & 92 \\
\hline $\mathrm{Sm}$ & 103 & 98 & 85 & 101 & 85 & 86 & 91 & 98 & 88 & 113 & 85 & 89 \\
\hline $\mathrm{Eu}$ & 104 & 97 & 96 & 98 & 104 & 97 & 91 & 83 & 94 & 97 & 98 & 100 \\
\hline Gd & 109 & 96 & 108 & 111 & 98 & 97 & 102 & 99 & 89 & 105 & 90 & 100 \\
\hline $\mathrm{Tb}$ & 97 & 107 & 109 & 132 & 71 & 92 & 67 & 83 & 72 & 87 & 67 & 87 \\
\hline Dy & 81 & 94 & 83 & 98 & 73 & 94 & 69 & 85 & 80 & 100 & 71 & 95 \\
\hline Ho & 86 & 105 & 72 & 91 & 72 & 83 & 66 & 85 & 70 & 93 & 63 & 75 \\
\hline $\mathrm{Er}$ & 72 & 97 & 69 & 104 & 73 & 99 & 76 & 94 & 76 & 100 & 69 & 97 \\
\hline $\mathrm{Tm}$ & 69 & 107 & 45 & 79 & 61 & 79 & 71 & 93 & 70 & 78 & 48 & 76 \\
\hline $\mathrm{Yb}$ & 70 & 96 & 64 & 106 & 65 & 104 & 67 & 87 & 73 & 98 & 64 & 95 \\
\hline $\mathrm{Lu}$ & 73 & 107 & 63 & 97 & 59 & 90 & 64 & 88 & 71 & 98 & 54 & 83 \\
\hline
\end{tabular}

A: Acid Digestion Method.

B: Fusion Method.

The lanthanum recovery for the sediment samples was found better using the fusion method in comparison to the acid digestion method. The results showed that the recovery of the heavier rare earth elements were non-uniform for the acid dissolution technique.

The recovery of lanthanides for the soil samples follows a similar trend as for the sediment samples. But in the case of lanthanum, acid recovery was observed to be better than fusion recovery. Heavier rare earth element recovery is not only non-uniform using the acid digestion technique but is also much less in comparison to the fusion technique. 
The percentage relative standard deviation (standard deviation $\mathbf{x}$ $100 /$ mean concentration) for the determination of the lanthanides was less than $4 \%$, confirming good precision of these methods. The limit of quantitation (LOQ, 10 times the standard deviation of the digestion procedure blank solution) was expressed as the concentration in the samples, thereby accounting for the dilution factor used. The LOQ observed for the lanthanides was in ppb level using the fusion method. Though the observed LOQ in the acid dissolution technique was lower than the fusion method, the recovery of HREEs was nonuniform.

\section{CONCLUSION}

The fusion technique using a mixture of lithium metaborate and tetraborate in the ratio of 35:65 allowed complete and uniform extraction of all of the lanthanides from soil and sediment samples. The advantage of the mixture of lithium metaborate and lithium tetraborate in the above ratio was in lowering the fusion temperature. The extraction for LREEs using the acid dissolution technique is very good, whereas for the HREEs it is incomplete. The recovery of lanthanum from soil samples was better than from sediment samples.

The present study established that the fusion technique is superior to acid dissolution. Acid dissolution is good for only LREEs of soil and sediment samples. The fusion technique for soil and sediment samples using lithium metaborate and lithium tetraborate followed by ICP-MS measurement is a simple, rapid, less strenuous, precise, and accurate method for the determination of REEs. The LOQ for lanthanides using the fusion method was in the 30 to $100 \mathrm{ppb}$ range and for the acid dissolution technique it was in the 15 to $50 \mathrm{ppb}$ range.

\section{ACKNOWLEDGMENT}

The authors are thankful to the Director General, Geological Survey of India, for giving permission to publish this work.

Received March 2, 2016.

Revision received June 3, 2016.

\section{REFERENCES}

1. B. Merkert, Phytochem. 26(12), 3167 (1987).

2. P. Henderson, General geochemical properties and abundances of the rare earth elements. Rare earth element geochemistry (P. Henderson ed.), Elsevier, New York, USA, 2 (1984).

3. M. Ravichandran, Mar. Pollut. Bull. 32, 719 (1996).

4. G. Matisoff, M.E. Ketterer, C.G.Wilson, R.Layman, and P.J.Whiting, Environ. Sci. Technol. 35, 3356 (2001).

5. S.Wei, L. Puling, Y.Mingyi, and $X$. Yazhou, J. Rare Earths. 21, 587 (2003).

6. X.C.Zhang, M.A. Nearing, V.O. Polyakov, and J.M. Friedrich, Soil. Sci. Soc. Am. J. 67, 279 (2003).

7. I. Olmez, E.R. Sholkovitz, D. Hermann, and R.P. Eganhouse, Environ. Sci. Technol. 25, 310 (1991).

8. W.T. Holser, Palaeogeogr. Palaeoclimatol. Palaeoecol. 132, 309 (1997).

9. R.Ramesh, A.L Ramanathan, R. A James, V. Subramanian, S.B. Jacobsen and H.D Holland, Hydrobiologia 397, 89 (1999).

10. G.E. Webb, and B.S. Kamber, Geochim. Cosmochim. Acta 64, 1557 (2000).

11. Y. Nozaki, D. Lerche, D.S. Albibo, and M. Tsutsumi, Geochim. Cosmochim. Acta 64, 3975 (2000)

12. S.Y. Yang, H.S. Jung, M.S. Choi, and C.X. Li, Earth Planet. Sci. Lett. 201, 407 (2002).

13. Y. I. Lee, H.S. Lim, and H.I. Yoon, Geochim. Cosmochim. Acta 68, 4319 (2004).
14. V.G. Caccia and F.J. Millero, Mar. Chem. 104, 171 (2007).

15. K. Tanaka, F. Akagawa, K. Yamamoto, Y. Tani, I. Kawabe, and T. Kawai, Quaternary Sci. Rev. 26, 1362 (2007).

16. T.T. Chao and R.F Sazolone, J. Geochem. Explor. 44, 65 (1992).

17. W. Doherty and A. Vander Voet, Can. J. Spectrosc. 30, 135 (1985).

18. A.R. Date and D. Hutchinson, J. Anal. At. Spectrom. 2, 269 (1987).

19. K.E. Jarvis, Chem. Geol. 68, 31 (1988).

20. M.B. Shabani and A. Masuda, Anal. Chem. 63, 1 (1991).

21. J.G. Sengupta, Talanta 31, 1045 (1984).

22. J.G. Sengupta, Talanta 32, 1 (1985).

23. J.G. Sengupta, Talanta 8, 93 (1993)

24. G.N. Eby, Anal. Chem. 44, 2137 (1972).

25. N.N. Fedyunina, K. B. Ossipov, I.F. Seregina, M.A. Bolshov, M.A. Statkus, and G.I. Tsysin, Talanta 102, 128 (2012).

26. F. Claisse, "Fusion and Fluxes", Comprehensive Analytical Chemistry: Sample Preparation for Trace Element Analysis, Vol. 41, Elsevier, pp. 301-311 (2003).

27. J.G. Sengupta, Talanta 42, 269 (1995)

28. Z. Begum, V.Balaram, S.M Ahmad, M. Satyanarayanam, and T. Gnaneshwar Rao, At. Spectrosc. 28(2), 41 (2007).

29. B.Zawisza, K. Pytlakowska, B. Feist, M. Polowniak, A.Kita, and R.Sitka, J. Anal. At. Spectrom. 26, 2373 (2011).

30. V. Balaram, Tr. Anal. Chem. 15, 475 (1996).

31. L. Haichen, L. Ying, and Z. Zhanxia, Spectrochim. Acta, Part B, 53(10), 1399 (1998).

32. K. Shinotsuka and M. Ebihara, Anal. Chim. Acta 338(B), 237 (1997). 